\title{
Fluorescence Enhancement of a Dicyanostilbene Derivative Film Casted from an Alcoholic Solution Triggered by UV light Irradiation
}

\author{
Jun-ichi Fujimori, ${ }^{1}$ Akio Katsuki, ${ }^{2}$ and Fuyuki Ito* ${ }^{1}$ \\ ${ }^{1}$ Department of Chemistry, Institute of Education, Shinshu University, 6-ro, Nishinagano, Nagano 380-8544, Japan \\ ${ }^{2}$ School of General Education, Shinshu University, 3-1-1 Asahi, Matsumoto 390-8621, Japan
}

(Received $<$ Month $><$ Date $>$, $<$ Year $>$; CL- $<$ No $>$; E-mail: $<$ fito@shinshu-u.ac.jp $>$ )

The films of a dicyanostilbene derivative (DBDCS) cast from an alcoholic solution show aggregation-induced emission enhancement by UV light irradiation and a fluorescence color change from green to blue by heating. The former can be attributed to the planarization by rotational motion around the olefin moieties due to an elevation in the local temperature via the excited-state by UV irradiation. The later originates from the phase transformation of DBDCS with cooperative alcoholic desorption and molecular reorganization.

Polymorphism in organic crystals has been a fundamental issue in organic solid chemistry, which is strongly related to the physical and chemical properties. ${ }^{1,2}$ Aspirin $^{\circledR}$ (acetylsalicylic acid) as a famous painkiller, for example, has two types of polymorphisms. ${ }^{2}$ It is known that its efficacy strongly depends on the polymorphism, ${ }^{3}$ because of the solubility depending on the molecular stacking in the crystal. Regarding material science, there are polymorphic crystals with different properties of crystal color ${ }^{4}$ or fluorescence color. ${ }^{5,6}$ In these ways, the polymorphism shows the physicochemical properties of organic crystals leading to various functionalities such as electric conductivity and emission properties, depending on the molecular stacking. ${ }^{7,8}$ It is suggested that the control of the polymorphism is important to providing the bioavailability of pharmaceutical products or color tuning of the organic light emissive materials in the solid state. Although the molecular structure itself can be designed relatively easily, it is hard to control the crystallization condition for selectively getting the desired polymorph. Therefore, it is important to control the polymorphism and to understand the crystal formation/growth process in order to develop the crystal engineering.

We have reported the crystal formation and growth process of a cyanostilbene derivative $(\mathrm{CN}-\mathrm{MBE})^{9},{ }^{10}$ with aggregation-induced emission enhancement (AIEE) ${ }^{11}$ during solvent evaporation. ${ }^{12}$ The fluorescence intensity of the droplet was weak just after the dropping. The fluorescence intensity gradually increased with a time associated with the solvent evaporation. We found that J-aggregates act as precursors for crystal nuclei and that the growth from the Jaggregates to crystal nuclei is the rate-determining step in the nucleation process. The methodologies discussed here can be used to understand the growth kinetics from monomer to crystal via the crystal nuclei, which are required for organic crystal growth in real time. By using a molecule having properties both of polymorphism and AIEE phenomena, we can get more detail information about the mechanism and kinetics of the appearance of the polymorphs during the crystal formation process. Regarding this point, we focused on

(2Z,2'Z)-2,2'-(1,4-phenylene)bis(3-(4- butoxyphenyl)acrylonitrile) (DBDCS, Figure 1) as a molecule with a polymorphism (green-emitting (G-phase) and blueemitting (B-phase)) and AIEE properties, which are originated from the difference between $\pi-\pi$ overlapping via $\mathrm{C}-\mathrm{H} \cdots \mathrm{N}$ and $\mathrm{C}-\mathrm{H} \cdots \mathrm{O}$ hydrogen bonding and aggregationinduced planarization, respectively. ${ }^{5}, 13$ The metastable Gphase and the thermodynamically favored B-phase shows green and blue emission, respectively. We have found that the as-cast DBDCS film prepared from an alcoholic solvent shows no emission. The green fluorescence significantly increased by $365 \mathrm{~nm}$ UV light irradiation. In this study, to clarify the AIEE behavior, we measured the fluorescence and IR spectra during the fluorescence changes of the DBDCS film.

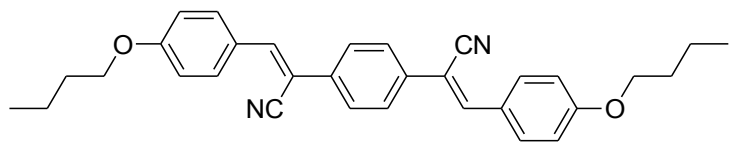

Figure 1. Chemical structure of DBDCS.

DBDCS was synthesized according to a previous report. ${ }^{13}$ The DBDCS film was prepared by drop-casting $1 \times 10^{-4} \mathrm{~mol} \mathrm{dm}^{-3}$ in ethanol onto a cover glass, then drying at room temperature under vacuum for $24 \mathrm{~h}$. In order to measure the IR spectra using the Attenuated Total Reflection (ATR) method, the film was cast on $\mathrm{ZnSe}$ substrates from DBDCS in an ethanol solution. The fluorescence spectra were recorded by a USB4000 (Ocean Optics). An LED (Siokaze Engineering UVF-365 AC, $\lambda=365 \mathrm{~nm}, 0.03 \mathrm{~mW}$ ) was used as the excitation light source. The IR spectra were recorded by a Shimadzu IRAffinity fitted ATR device (Shimadzu ATR8200HA). All experiments were carried out at room temperature. To assess the optimized structures and vibrational mode of the DBDCS, a TD-DFT calculation was performed at the RB3LYP/6-31g(d) level of theory.

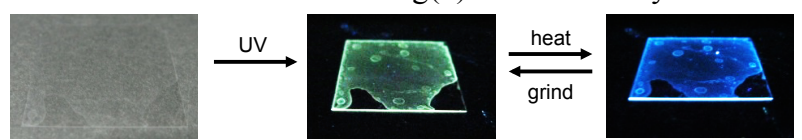

Figure 2. Fluorescence changes in a DBDCS film.

Figure 2 shows a photograph of the fluorescence change in the film by first UV irradiation at $298 \mathrm{~K}$, and then heating to $393 \mathrm{~K}$ on a hot plate. The film of DBDCS showed no fluorescence before the UV irradiation at $298 \mathrm{~K}$, which probably can be interpreted as hydrogen-bonding due to the residual ethanol in the films, even during the heat treatment of the film around the melting point of DBDCS $(446 \mathrm{~K})^{13}$. It is suggested that the hydrogen-bonding between DBDCS and ethanol is relatively strong. A green fluorescence from the film appeared during UV irradiation at $365 \mathrm{~nm}$. This green 
fluorescence turns blue by heating at $393 \mathrm{~K}$ on a hot plate, which is identical to the crystalline transformation temperature. ${ }^{13}$ Furthermore, this blue fluorescent returned to green by smearing with a spatula. The interconversion between the green (G-phase) and the blue (B-phase) originated from the amorphous-crystal phase transformation by the mechanical perturbation as reported by Park et al. ${ }^{13} \mathrm{We}$ first discovered the phenomena about the photoinduced fluorescence enhancement (AIEE) in the DBDCS films cast from an ethanol solution.

To clarify the phenomena, we measured the fluorescence spectral change of the DBDCS films after the UV irradiation. Figure 3a shows the fluorescence spectra of the DBDCS film as a function of time after the $365 \mathrm{~nm}$ light irradiation. A slight fluorescence of the DBDCS film showed just after the measurement. The fluorescent intensity increased with the increasing time of the UV irradiation, the peak of which was around $515 \mathrm{~nm}$. This peak is identical to the G-phase of DBDCS. ${ }^{14}$ It is indicated that the phase transformation occurred from the non-fluorescence state to the G-Phase in the films by the UV irradiation. The time evolution of the peak intensity at $515 \mathrm{~nm}$ is shown in Figure $3 \mathrm{~b}$. The fluorescence intensity monotonically increased then reached a constant value with time, indicating the photoinduced phenomena of the fluorescence enhancement.
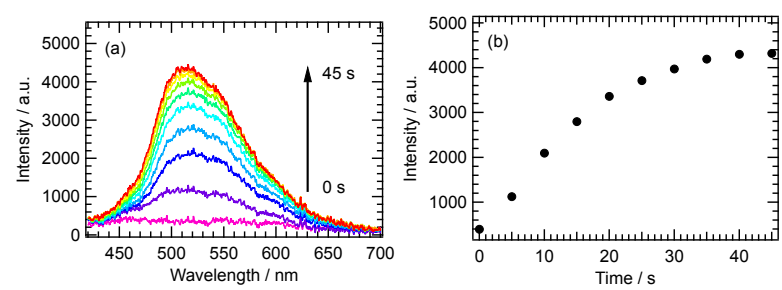

Figure 3. (a) Fluorescence spectral change in DBDCS film by the UV irradiation and (b) the time evolution of the peak intensity at $515 \mathrm{~nm}$.

In order to confirm the irradiation light intensity dependence of the fluorescence enhancement, Figure $4 \mathrm{a}$ shows the changes in the fluorescence intensity at $515 \mathrm{~nm}$ as a function of the irradiation time. The fluorescence intensity monotonically increased and reached a constant value, and then the intensity decreased. The decrease in the intensity most probably originated from the degradation or photochemical reaction such as cyclization reaction of DBDCS by the continuous photoirradiation. The rise time increased with increasing the UV intensity. Because the elementary reaction process is unclear, the time to reach half of the maximum intensity $\left(t_{1 / 2}\right)$ was initially used as an evaluation of the phenomena. The logarithmic $t_{1 / 2}$ values are a straight line versus the logarithmic relative light intensity with a slope of 3 as shown in Figure $4 \mathrm{~b}$. It is suggested that this phenomenon originates from a three-order reaction or related to three molecules triggered by the UV irradiation.
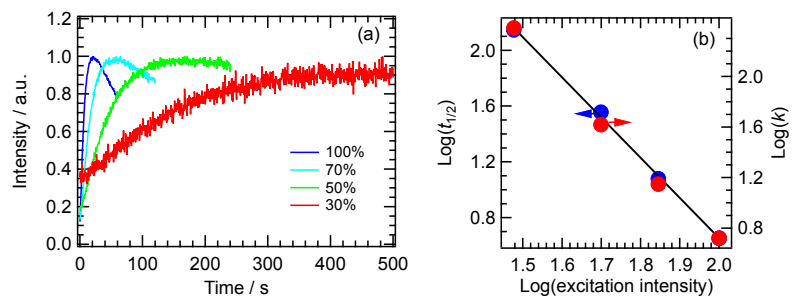

Figure 4. (a) Time course of the fluorescence intensity for irradiation light intensity of DBDCS film by the UV irradiation and (b) the log-log plot of the time to reach half of the maximum (blue circles) and time constant based on the modified Langmuir-Hill equation (red circles).

Table 1. The time constant and cooperative factor obtained by the modified Langmuir-Hill equation of the DBDCS fluorescence enhancement

\begin{tabular}{rcl}
\hline Intensity & $\alpha$ & \multicolumn{1}{c}{$k / \mathrm{s}$} \\
\hline $100 \%$ & $1.54 \pm 0.01$ & $5.26 \pm 0.1$ \\
$70 \%$ & $1.53 \pm 0.01$ & $14.1 \pm 0.2$ \\
$50 \%$ & $1.31 \pm 0.01$ & $41.6 \pm 0.5$ \\
$30 \%$ & $1.00 \pm 0.01$ & $242 \pm 6$ \\
\hline
\end{tabular}

The increasing irradiation light intensity dependence of the fluorescence suggests that the cooperative effect will relate to these changes. We evaluate the changes in the fluorescence intensity $(\eta(t))$ by using the following equation that is the modified Langmuir-Hill equation: ${ }^{14}$

$$
\eta(t)=\frac{t^{\alpha}}{t^{\alpha}+k^{\alpha}}
$$

where $t$ and $k$ are the time and time constant, respectively. $\alpha$ is a phenomenological exponential parameter (known as the Hill parameter), which aims at identifying whether the photoinduced fluorescence enhancement is a cooperative (if greater than 1), a noncooperative (if equal to 1), or a negatively cooperative (if less than 1) process. The fitting results, which are listed in Table 1, show the cooperative effect. The $k$ parameter represents a time constant that gives the time value at which the half occupation $\left(t_{1 / 2}\right)$ is reached, which is comparable to these values as shown in Figure 4b.

In order to confirm the molecular structure change, we measured the IR spectra of the DBDCS film on a $\mathrm{ZnSe}$ substrate before and after photoirradiation concomitant with the microcrystal powder of DBDCS, ethanol, and DFT calculation, which are shown in Figure 5. In the difference spectrum between before and after the UV irradiation (sky blue line), positive absorbances were observed at 1593, 1510, 1253 and $1176 \mathrm{~cm}^{-1}$. It is indicated that the positive absorbances become stronger and weaker in oscillator strength of the bands within the molecule by the photoirradiation. The bands of the positive absorbance were, respectively, assigned to the stretching band of the two olefins $\left(1593 \mathrm{~cm}^{-1}\right)$, stretching band of the benzene substituents $(1510$ $\left.\mathrm{cm}^{-1}\right)$, stretching band of the ether groups $\left(1253 \mathrm{~cm}^{-1}\right)$ and stretching vibration of the benzene rings $\left(1176 \mathrm{~cm}^{-1}\right)$ based on the vibrational analysis of the DFT calculations. Therefore, the spectral change by the photoirradiation can be ascribed to the conformational change around the olefin and the ether group. 


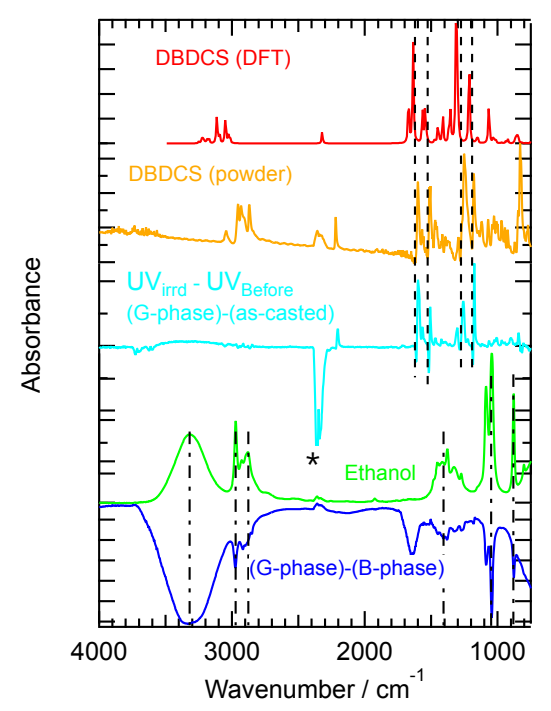

Figure 5. IR spectral change in DBDCS film due to the UV irradiation or heat. Asterisk indicates the band of $\mathrm{CO}_{2}$.

Next, we measured the IR spectral change associated with the thermochromic fluorescence change by heating of the DBDCS film. The difference spectra before and after the heating at $393 \mathrm{~K}$ corresponding to change from the G-phase to B-phase indicates the desorption of ethanol, which is shown by the blue line in Figure 5. The difference spectra strongly suggested that ethanol can evaporate by heating at $393 \mathrm{~K}$. It most probably originated from the small amount of ethanol in the film even after drying under vacuum because of the strong interaction of the hydroxy group on the glass substrate. Interestingly the heat-treated DBDCS film before UV irradiation does not show the blue emission, and the ethanol can easily desorb for DBDCS after UV irradiation. The Gphase film can transform to the B-phase by the heat-treatment according to the Ostwald rule of state. It is suggested that the UV irradiation acts the cooperative re-organization of the molecules due to the photo-thermal effects via the excitedstate.

The AIEE and fluorescence color change can be explained by following the scheme based on the IR spectra, which are shown in Figure 6. The as-casted film from the alcoholic solution forms a liquid-like or amorphous-like state of DBDCS including solvent molecule, which shows no emission due to the torsion or disordered form of DBDCS. It can be an analog with the fluorescence property of DBDCS in solution. The UV light irradiation produced a transformation from the non-emissive state to the G-phase. The IR spectra before and after the UV irradiation indicate the conformational change around the olefin and the ether group. The initial liquid-like state can reorganize and planarize so that the excited non-emissive species will mainly deactivate by a non-radiative path, leading to activating the rotational motion around olefin moieties and to an elevation of the local temperature (photo-thermal annealing). ${ }^{15}$ The phenyl rings in DBDCS of the G-phase form on the same plane due to the structural change by UV irradiation. This finding supports the results of the X-ray structure analysis of the G-phase. ${ }^{13}$ The change in the $\mathrm{C}-\mathrm{O}$ band in the ether group can be considered to be due to the hydrogen bond breaking between $\mathrm{H}$ (arene) $\cdots \mathrm{O}$ (ether). Interestingly the non-emissive films are fabricated only from the alcoholic solution. The following phase change from the G-phase to the B-phase causes the crystal phase transformation that is similar to the previous report ${ }^{13}$ concomitant with the desorption of ethanol from the film.

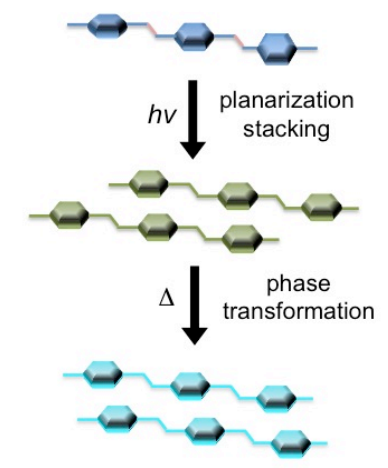

Figure 6. Schematic representation of fluorescence changes in the DBDCS film.

In conclusion, we have found that the AIEE of the DBDCS film are due to the cooperative reorganization by UV light irradiation via the excited-state. It was found that the process involves the planarization and phase transformation of the aggregated form of DBDCS. This result also provides applications for fluorescence-switching materials based on the phase transformation by both heat and photoirradiation.

This study was partly supported by Grant-in-Aid for Scientific Research (C) (No. 26410009) from JSPS and for Grant-in-Aid for Scientific Research on Innovative Areas "Photosynergetics" (No. 15H01081) from MEXT, Japan. JF was also supported by a Grant-in-Aid of the Nagano Society for the Promotion of Science.

\section{References and Notes}

1 J. Bernstein, Polymorphism in Molecular Crystals Oxford University Press, New York, 2008.

2 G. R. Desiraju, J. J. Vittal, A. Ramanan, Crystal Engineering A Textbook. World Scientific, Singerpore, 2011.

3 P. Vishweshwar, J. A. McMahon, M. Oliveira, M. L. Peterson, M. J. Zaworotko, J. Am. Chem. Soc. 2005, 127, 16802.

4 L. Yu, G. A. Stephenson, C. A. Mitchell, C. A. Bunnell, S. V. Snorek, J. J. Bowyer, T. B. Borchardt, J. G. Stowell, S. R. Byrn, J. Am. Chem. Soc. 2000, 122, 585

5 S. J. Yoon, S. Park, J. Mater. Chem. 2011, 21, 8338.

6 H. Ito, M. Muromoto, S. Kurenuma, S. Ishizaka, N. Kitamura, H. Sato, T. Seki, Nature Communications 2013, 4.

7 M. D. Curtis, J. Cao, J. W. Kampf, J. Am. Chem. Soc. 2004, 126, 4318.

8 R. J. Li, W. P. Hu, Y. Q. Liu, D. B. Zhu, Acc. Chem. Res. 2010, 43, 529.

9 B. K. An, D. S. Lee, J. S. Lee, Y. S. Park, H. S. Song, S. Y. Park, J. Am. Chem. Soc. 2004, 126, 10232.

10 T. Hirose, Y. Tsunoi, Y. Fujimori, K. Matsuda, Chem. Eur. J. 2015, $21,1637$.

11 Y. N. Hong, J. W. Y. Lam, B. Z. Tang, Chem. Soc. Rev. 2011, 40, 5361.

12 F. Ito, J.-i. Fujimori, CrystEngComm 2014, 16, 9779.

13 S. J. Yoon, J. W. Chung, J. Gierschner, K. S. Kim, M. G. Choi, D. Kim, S. Y. Park, J. Am. Chem. Soc. 2010, 132, 13675.

14 G. Recio, D. Gallach, M. Manso Silvan, K. Fukami, R. J. Martin Palma, G. Rafael Castro, A. Munoz-Noval, J. Phys. Chem. C 2014, $118,14905$.

15 F. Ito, H. Sato, Y. Ugachi, N. Oka, S. Ito, H. Miyasaka, Photochem. Photobiol. Sci. 2015, 14, 1896. 